\title{
Novel focal adhesion kinase 1 inhibitor sensitizes lung cancer cells to radiation in a p53-independent manner
}

\author{
SEUNG-YOUN JUNG ${ }^{1 *}$, SEONGHO KHO ${ }^{1 *}$, KYUNG-HEE SONG $^{1}$, JIYEON AHN $^{1}$, IN-CHUL PARK ${ }^{2}$, \\ KY-YOUB NAM ${ }^{3}$, SANG-GU HWANG ${ }^{1}$, SEON-YOUNG NAM ${ }^{4}$, SEONG-JUN CHO ${ }^{1,4}$ and JIE-YOUNG SONG ${ }^{1}$ \\ ${ }^{1}$ Division of Applied Radiation Bioscience, ${ }^{2}$ Division of Basic Radiation Bioscience, \\ Korea Institute of Radiological and Medical Sciences, Seoul 01812; ${ }^{3}$ Paros I\&BT Co., Ltd., 1408, 38 \\ Heungan-daero 427 beon-gil, Dongan-gu, Anyang-si, Gyeonggi-do 14059; ${ }^{4}$ Low-Dose Radiation Research Team, \\ Radiation Health Institute, Korea Hydro and Nuclear Power Co., Ltd., Seoul 01450, Republic of Korea
}

Received June 22, 2017; Accepted September 25, 2017

DOI: $10.3892 /$ ijo.2017.4141

\begin{abstract}
Focal adhesion kinase 1 (FAK1) is known to promote tumor progression and metastasis by controlling cell movement, invasion, survival and the epithelial-to-mesenchymal transition in the tumor microenvironment. As recent reports imply that FAK1 is highly associated with tumor cell development and malignancy, the inhibition of FAK1 activity could be an effective therapeutic approach for inhibiting the growth and metastasis of tumor cells. In this study, we aimed to determine the effect of a novel synthetic FAK1 inhibitor 2-[2-(2-methoxy-4-morpholin-4-yl-phenylamino)5-trifluoromethyl-pyrimidin-4-ylamino]- $N$-methyl-benzamide, (MPAP) on lung cancer cells. MPAP suppressed cancer cell proliferation and the phosphorylation of FAK1. Combined treatment with MPAP and irradiation (IR) showed enhanced suppression of cancer cell proliferation in wild-type p53 cells and more intense suppression in p53-null cells. In addition, the combination treatment effectively induced G1 cell cycle arrest in a p53-independent manner. In an in vivo tumor xenograft mouse model, treatment with both MPAP and IR reduced tumor growth more than the treatment with IR or MPAP alone. Overall, these data demonstrate that the radiosensitizing effect of MPAP is mediated by the regulation of retinoblastoma protein $(\mathrm{RB})$ phosphorylation in a p53-independent manner.
\end{abstract}

Correspondence to: Dr Jie-Young Song or Dr Seong-Jun Cho, Division of Applied Radiation BioScience, Korea Institute of Radiological and Medical Sciences, 75 Nowon-ro, Nowon-gu, Seoul 01812, Republic of Korea

E-mail: immu@kirams.re.kr

E-mail: sjuncho@kirams.re.kr

*Contributed equally

Key words: FAK1, radiosensitivity, cell cycle, cyclin D1, pRb

\section{Introduction}

Focal adhesion kinase 1 (FAK1) is a non-receptor tyrosine kinase encoded by the protein tyrosine kinase 2 (PTK2) gene and a multifunctional regulator of cell signaling for diverse cellular processes, including cell migration, growth factor signaling, cell cycle progression and cell survival $(1,2)$. FAK1 and proline-rich tyrosine kinase 2 (PYK2, a.k.a. FAK2) are of the FAK family, and intensive studies of FAK1 have demonstrated its role in tumor malignancy. FAK1 was initially identified as a protein tyrosine kinase that is phosphorylated in response to local cell attachment, and it was recently found to be functionally phosphorylated at Tyr residues 397, 576 and 577 via interactions with integrins or other growth factor receptors (1-3). In particular, the phosphorylation of Tyr397 is known to lead to the binding and activation of $\mathrm{SH} 2$ domain proteins, such as Src family proteins, phosphoinositide 3-kinase, phospholipase C- $\gamma$, Grb7 and SH3 domain proteins, to transduce the signals for cell adhesion/migration, actin polymerization, and cell survival $(4,5)$.

In the tumor microenvironment, FAK1 is known to promote the tumor progression and metastasis by controlling the cell movement, invasion, survival and gene expression for the epithelial-to-mesenchymal transition (6). In fact, data from the Cancer Genome Atlas database show that the expression of $P T K 2$ mRNA is elevated in approximately $37 \%$ of serious ovarian tumors and $26 \%$ of invasive breast cancers, and these increased levels are correlated with poor overall survival (7-9). In addition, FAK1 is reported to be overexpressed in many malignant tumors, including nonsmall cell lung cancers, exerting an oncogenic effect $(10,11)$. These reports imply that FAK 1 is highly associated with tumor cell development and malignancy and that the inhibition of FAK1 activity may be an effective therapeutic approach for inhibiting the growth and metastasis of tumor cells.

Currently, small molecule FAK1 inhibitors such as TAE226, PF-562, 271 and GSK-2256098 are being developed as promising chemotherapeutic agents to prevent tumor growth, metastasis, vascular permeability and angiogenesis in mouse models (12-21). Among these inhibitors, TAE226 
appears to inhibit FAK1 $\left(\mathrm{IC}_{50}, 5.5 \mathrm{nM}\right)$ as well as other protein tyrosine kinases such as PYK2, insulin receptor, insulin-like growth factor-I receptor and c-Met (21). TAE226 suppresses cell proliferation and invasion and promotes cell death in glioma and ovarian tumor models $(17,22,23)$. In combination with docetaxel, a microtubule stabilizer, TAE226 significantly decreases angiogenesis and invasion in ovarian cancer $(17,22,23)$. In our previous study, we synthesized a new small molecule inhibitor of FAK1, 2-[2-(2-methoxy-4-morpholin-4-yl-phenylämino)-5-trifluoromethyl-pyrimidin-4-ylamino]- $N$-methyl-benzamide (hereafter abbreviated as MPAP), which blocks the phosphorylation of Tyr397 by modifying a functional group on the bis-anilino pyrimidine moiety of TAE226, increasing hydrophobic interactions with the side-chain of the gatekeeper residue Met499. This inhibitor exhibits potent kinase inhibition of FAK1, with an $\mathrm{IC}_{50}$ value of $4.8 \mathrm{nM}$, which is lower than that of TAE226 (24). Therefore, in this study, we further investigated the antitumor activity of the novel FAK1 inhibitor, MPAP, on lung cancers as well as determined its effect when combined with radiation, which is the standard treatment for patients with lung cancer.

\section{Materials and methods}

Cell lines and cell culture. Human lung carcinoma cell lines (A549 and H1299) and human normal lung fibroblast cell lines (IMR90 and WI38) were obtained from the American Type Culture Collection (ATCC; Manassas, VA, USA). All cell lines were cultured in RPMI-1640 (Life Technologies, Carlsbad, CA, USA) supplemented with $10 \%$ fetal bovine serum (FBS; Life Technologies) and maintained at $37^{\circ} \mathrm{C}$ in a humidified $5 \%$ $\mathrm{CO}_{2}$ atmosphere.

Irradiation (IR). Cells were uniformly irradiated at room temperature with various doses of a ${ }^{137} \mathrm{Cs} \gamma$-source (Gammacell 3000 ELan; MDS Nordion, Ottawa, ON, Canada) at a dose rate of $3.25 \mathrm{~Gy} / \mathrm{min}$. Cells of the control group were simultaneously exposed to sham IR. Tumor xenograft mice were irradiated with a ${ }^{60} \mathrm{Co} \gamma$-source using the Theratron 780 (MDS Nordion) radiotherapy unit when the tumor volume reached $\sim 100-200 \mathrm{~mm}^{3}$. During IR, mice were covered with a $0.5-\mathrm{cm}$ bolus and exposed to a single dose of $5 \mathrm{~Gy}$ at a dose rate of $0.5296 \mathrm{~Gy} / \mathrm{min}$.

MTT assay. Cells $\left(1 \times 10^{6}\right)$ were seeded in 6-well plates and incubated for $24 \mathrm{~h}$ after various treatments. MPAP was purchased from 4Chem Laboratory Co., Ltd. (Suwon, Korea). Then, $5 \mathrm{mg} / \mathrm{ml}$ of MTT was added to the cell culture medium, and cells were further incubated for 2-4 h. After the supernatant was removed, dimethyl sulfoxide (DMSO) was added to the cells and mixed by shaking for $15 \mathrm{~min}$. Cell viability was determined by measuring the absorbance at $570 \mathrm{~nm}$ using a microplate reader.

Colony formation assay. Cells (500/well) were seeded in a 6-well plate and cultured under various conditions for 12 days. Cells were fixed and stained with $1 \%$ methylene blue in absolute methanol solution for 10 min. Colony density was quantified by densitometry.
Cell cycle analysis. Cells were harvested and then fixed in $75 \%$ ethanol for $1 \mathrm{~h}$. Cells were then washed with phosphate-buffered saline (PBS) and resuspended in PBS buffer containing $100 \mathrm{mg} / \mathrm{ml}$ RNase A (Sigma-Aldrich, Darmstadt, Germany) and $50 \mathrm{mg} / \mathrm{ml}$ propidium iodide (PI; Life Technologies), followed by incubation at $37^{\circ} \mathrm{C}$ for $30 \mathrm{~min}$. The percentage of cells in each cell cycle stage (G1/G0, S or G2/M) was analyzed with a FACSCalibur cell analyzer (BD Biosciences, San Jose, CA, USA) and FlowJo software version 10.1r5.

Western blot analysis. Cells were harvested and washed with ice-cold PBS. Whole cell lysates were prepared using $2 \mathrm{X}$ SDS/Sample buffer. Samples were then separated by $8-12 \%$ SDS-PAGE, transferred to a nitrocellulose membrane, and probed with the indicated antibodies, followed by ECL detection. The antibodies used in the present study were FAK1 (D2R2E; Cell Signaling Technology, Danvers, MA, USA), pFAK1 (T397) (D20B1; Cell Signaling Technology), pFAK1 (T576/577) (Cell Signaling Technology), pFAK1 (T925) (Cell Signaling Technology), pSrc (T416) (Cell Signaling Technology), RB (4H1; Cell Signaling Technology), phosphoRB (S807/811) (D20B12; Cell Signaling Technology), p53 (DO-1, Santa Cruz Biotechnology, Dallas, TX, USA), p21 (12D1; Cell Signaling Technology), cyclin D1 (92G2; Cell Signaling Technology), CDK4 (K9G3E; Cell Signaling Technology) and $\beta$-actin (Sigma-Aldrich).

Xenograft tumor model. Mice were maintained in an animal facility, and all animal experiments were performed according to the Korean National Guidelines of Laboratory Animal Experiments; protocols were approved by the Institutional Animal Care and Use Committee of the Korea Institute of Radiological and Medical Sciences. BALB/c nude mice (CAnN.Cg-Foxn $I^{\text {nu }} /$ CrljOri, 6 weeks old, female) were purchased from Orient Bio Inc. (Gyunggi-do, Korea). A549 cells $\left(1 \times 10^{6}\right)$ were injected subcutaneously into BALB/c nude mice. When the tumor volume reached an average of 100-200 $\mathrm{mm}^{3}$, MPAP was intraperitoneally introduced once at $10 \mathrm{mg} / \mathrm{kg}$ with or without IR at a dose of $5 \mathrm{~Gy}$. Tumor size was measured periodically 2-3 times per week. Tumor volume was calculated according to following formula: Tumor volume $\left(\mathrm{mm}^{3}\right)=(\text { width })^{2} \mathrm{x}$ length $/ 2$.

Statistical analysis. Statistical significance was determined by the analysis of variance (ANOVA). Non-parametric analyses using the Tukey's multiple comparison test were used when appropriate. A $\mathrm{P}<0.05$ was considered significant. Data are presented as means \pm SD (standard deviation) of three independent experiments.

\section{Results}

A new FAK1 inhibitor, MPAP, decreases the viability of lung cancer cells. MPAP was synthesized by modifying the functional group of TAE226 on the bis-anilino pyrimidine moiety with CF3, as described in a previous report (24) (Fig. 1A). First, we investigated whether MPAP reduces the activation state of the FAK1 protein and suppresses the growth of lung cancer cells. Treatment with MPAP sharply decreased the viability of A549 and H1299 lung cancer cells, with above 10- 
A

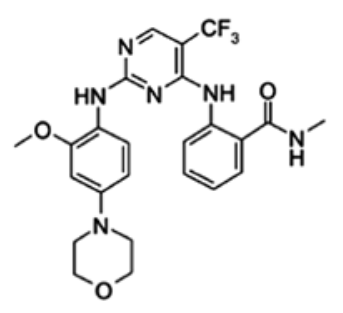

C

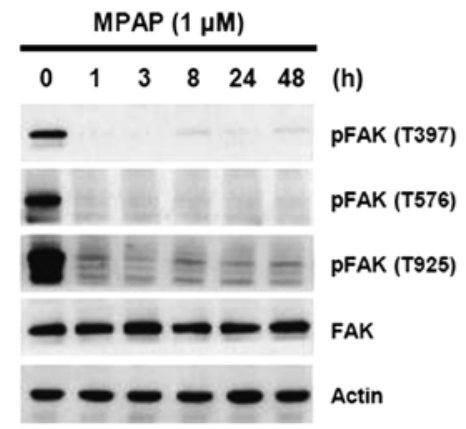

A549
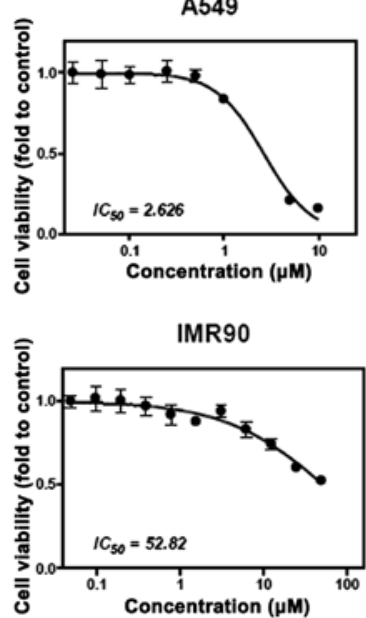

H1299

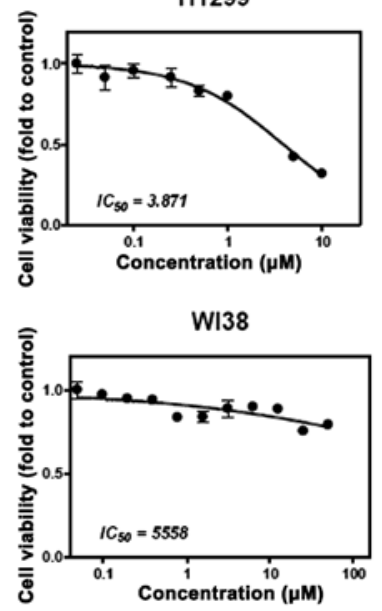

D

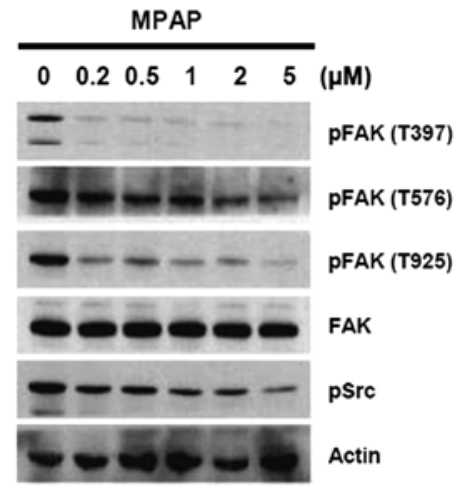

Figure 1. MPAP suppresses the phosphorylation of FAK1 and decreases the viability of A549 lung cancer cells. (A) Chemical structure of MPAP. (B) Effect of MPAP on viability of lung cancer cells (A549 and H1299) and normal lung fibroblast cells (IMR90 and WI38). The cells were treated with various doses of MPAP for $24 \mathrm{~h}$. Cell viability was determined by MTT assay. (C) A549 cells were treated with MPAP for various periods of time. Whole cell lysates were collected, and levels of total or phosphorylated FAK1 and actin were determined by western blot analysis. (D) A549 cells were treated with various doses of MPAP. Whole cell lysates were prepared, and levels of phospho-FAK1, FAK1, phospho-Src and actin were determined by western blot analysis.

to 1000 -fold higher sensitive than the viability of normal lung fibroblast cells (Fig. 1B). The $\mathrm{IC}_{50}$ value of MPAP on A549 and $\mathrm{H} 1299$ cancer cell viability is 2.626 and $3.871 \mu \mathrm{M}$, respectively. We then investigated the treatment time and dose of MPAP required to inhibit FAK efficiently for further experiments. As shown in Fig. 1C, $1 \mu \mathrm{M}$ MPAP markedly inhibited the phosphorylation of FAK1 at tyrosine residues 397, 576/577 and 925, and this lasted for $48 \mathrm{~h}$; in contrast, expression levels of FAK1 were not altered. In addition, FAK1 phosphorylation was also clearly inhibited by MPAP even at low concentrations $(0.2$ and $0.5 \mu \mathrm{M}$ ) that did not inhibit cell viability (Fig. 1D). Therefore, we applied the dose $0.2 \mu \mathrm{M}$ in the subsequent experiment to examine the combinatorial effect of MPAP with IR.

MPAP enhances radiation-induced clonogenic inhibition in lung cancer cells. To determine the radiosensitizing potential of MPAP in lung cancer cells, a colony formation assay was performed with $0.2 \mu \mathrm{M}$ MPAP in the presence or absence of a 4-Gy dose of IR. In this experiment, we used two different human lung cancer cell lines, A549 (wild-type p53) and H1299 (p53-null). In A549 cells, MPAP or IR alone markedly decreased colony formation, while complete inhibition was observed with combined MPAP and IR treatment, indicating that MPAP has a radiosensitizing effect (Fig. 2A and B, left panel). Similarly, MPAP or IR alone significantly decreased colony formation in H1299 cells, but the extent of inhibition was lower than that in A549 cells. Notably, MPAP combined with IR also completely inhibited colony formation in H1299 cells (Fig. 2A and B, right panel), suggesting that the combined effect of IR and MPAP was higher than that noted in the case of MPAP alone. These data suggest that MPAP has a radiosensitizing effect that is exhibited in a p53-independent manner.

Combined MPAP and IR treatment induces G1 arrest. To further investigate how MPAP and IR suppress tumor cell growth, we performed cell cycle analysis to assess cell cycle arrest and apoptosis. Apoptosis was not observed in any of the three treatments (MPAP, IR or combination) in either A549 or H1299 cells; this is because we used low doses of MPAP and IR to determine the combinatorial effect, avoiding direct toxicity. In A549 cells, MPAP treatment slightly increased the percentages of cells in the S and G2 phases, but IR alone did not affect the cell cycle distribution (Fig. 3A). The combination treatment of MPAP and IR significantly decreased the percentage of $S$ cells and arrested cells in the G1 and G2/M phases (Fig. 3A). Unlike in A549 cells, MPAP treatment clearly increased the G1 cell population in H1299 cells, while the combination treatment of MPAP and IR resulted in an even 
A

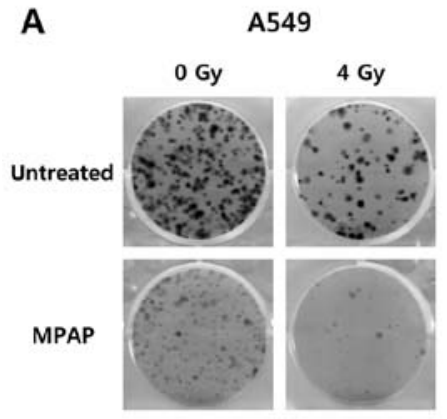

B

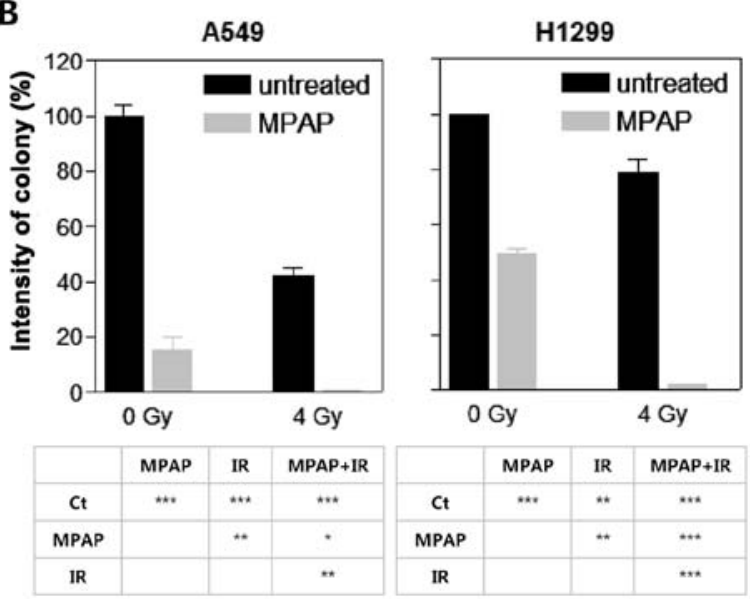

Figure 2. MPAP enhances radiation-induced growth inhibition of A549 and H1299 cells. A549 and H1299 cells were treated with $0.2 \mu \mathrm{M}$ of MPAP for $3 \mathrm{~h}$ and exposed to $4 \mathrm{~Gy}$ of $\gamma$-radiation, followed by incubation for 10 days. (A) A colony formation assay was then performed. (B) The density in each well (in A) was quantified, and the rate of colony formation under control conditions (untreated/0 Gy) was set at $100 \%$.

A
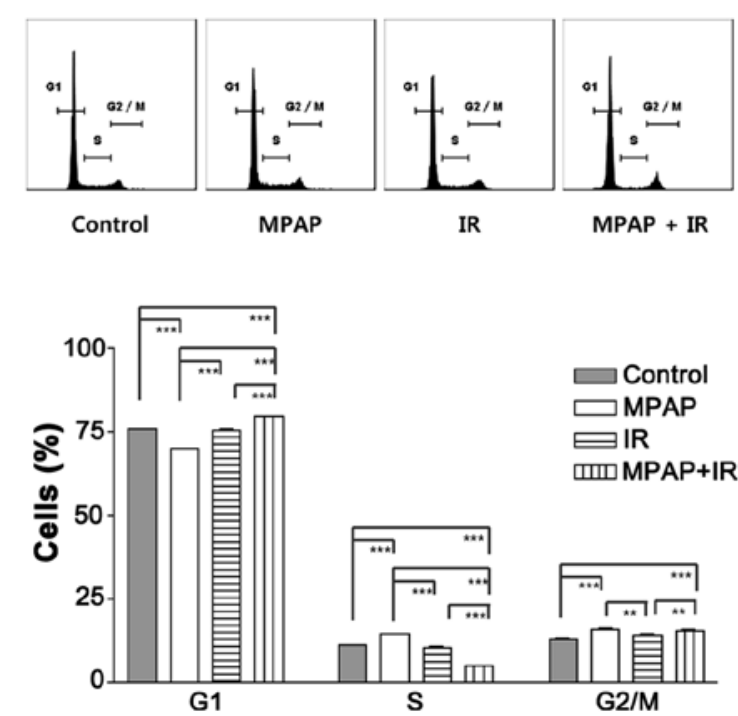

B
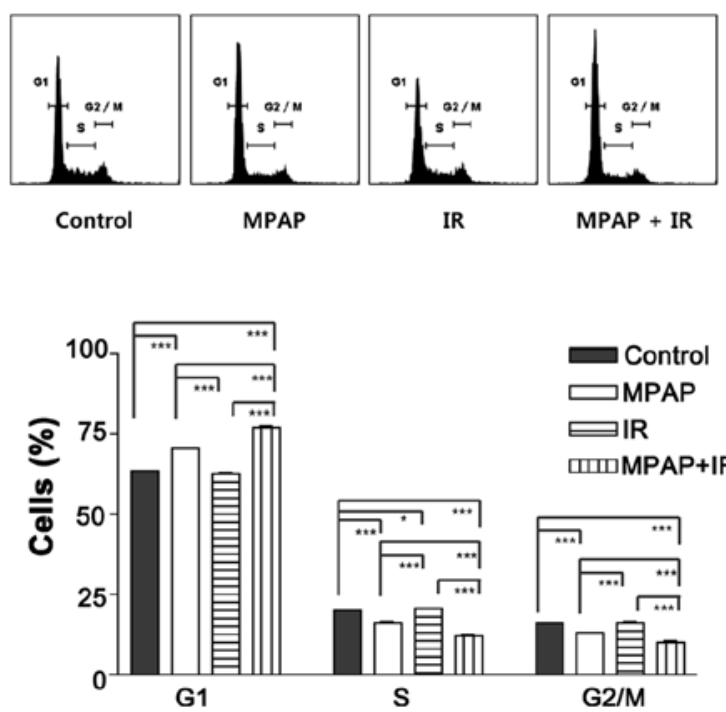

Figure 3. MPAP and IR induce G1 cell cycle arrest in a p53-dependent manner. (A) A549 or (B) H1299 cells were treated with $0.2 \mu \mathrm{M}$ of MPAP for 3 h and exposed to $4 \mathrm{~Gy}$ of $\gamma$-radiation, followed by incubation for $24 \mathrm{~h}$ and cell cycle analysis. Bottom panel shows percentages of cells in each cell cycle. Data are presented as means $\pm \mathrm{SD} ; \mathrm{n}=3 .{ }^{*} \mathrm{P}<0.05,{ }^{* *} \mathrm{P}<0.01$ and ${ }^{* * *} \mathrm{P}<0.001$ indicate statistically significant differences between experimental groups.

stronger increase in the G1 cell population (Fig. 3B). These data suggest that combined treatment with MPAP and IR significantly induces G1 cell cycle arrest via a p53-independent pathway.

Decreased cyclin D1/CDK4/pRB induces p53-independent Gl arrest by MPAP and IR. To determine which cell cycle regulators are involved in MPAP/IR-mediated cell cycle perturbation, we performed western blot analysis using antibodies for G1 cell cycle-related proteins. Consistent with the results of Fig. 1C and D, MPAP successfully inhibited the phosphorylation of FAK1 and decreased the levels of CDK4 and cyclin D1, key regulators of the G1-to-S phase progression of the cell cycle. The expression levels of cyclin D1 and CDK4 were markedly increased by IR treatment of A549 cells with wild-type p53 but were diminished by combined treatment with MPAP and IR. In addition, the expression of a major G1 checkpoint protein, RB, was also decreased by IR and decreased to an even greater extent by combined treatment with MPAP and IR in both cell lines. Levels of phosphorylation of RB at serine residues 807 and 811 exhibited the same pattern as RB expression (Fig. 4).

Considering that the p53/p21 pathway is a strong regulator of G1 cell cycle arrest and an upstream regulator in the cyclin D1/CDK4/RB signaling axis, levels of p53 and p21 proteins were determined. The expression levels of p53 and p21 were increased by IR in A549 cells. Notably, p21 expression was markedly increased by the combination of MPAP and IR in H1299 p53-null cells. These data suggest that MPAP/IR-mediated G1 cell cycle arrest may be attributed to downregulation of the cyclin D1/CDK4/pRB pathway rather than the p53/p21 pathway. 


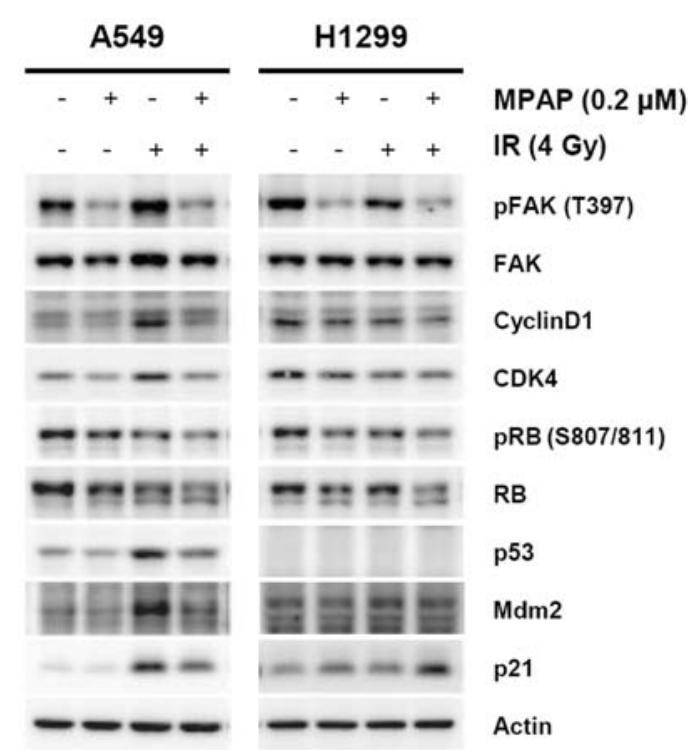

Figure 4. The regulation of cell cycle regulators for G1/S progression by combined treatment with MPAP and IR. A549 or H1299 cells were treated with $0.2 \mu \mathrm{M}$ of MPAP for $3 \mathrm{~h}$ and exposed to $4 \mathrm{~Gy}$ of $\gamma$-radiation, followed by incubation for $24 \mathrm{~h}$. Whole cell lysates were prepared, and levels of phospho-FAK1 (T397), FAK1, phospho-RB (S807/811), RB, p53, Mdm2, p21, cyclin D1, CDK4 and actin proteins were determined by western blot analysis.

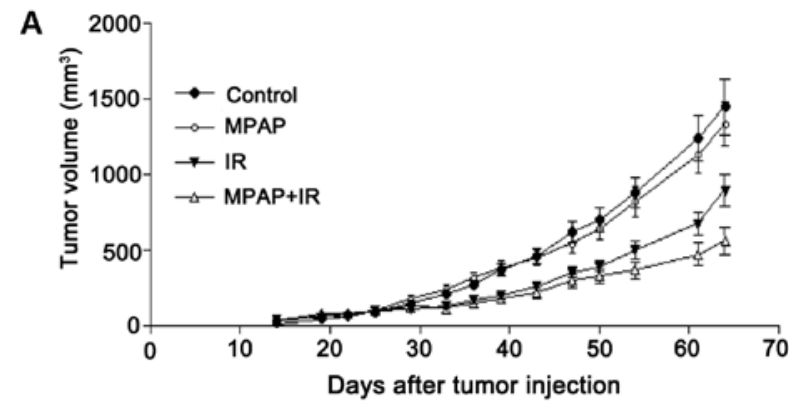

B

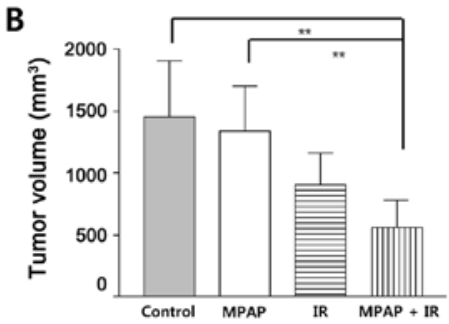

Figure 5. MPAP increases the antitumor effect of radiation in vivo. (A) A549 cells $\left(1 \times 10^{6}\right)$ were subcutaneously injected into the right thighs of BALB/c $\mathrm{nu} / \mathrm{nu}$ female mice. After the volumes of the tumors reached $100-200 \mathrm{~mm}^{3}$, mice were intraperitoneally injected with $10 \mu \mathrm{g} / \mathrm{kg}$ of MPAP with or without a local dose of 5 Gy of ${ }^{60} \mathrm{Co} \gamma$-ray IR. Tumor volume was evaluated periodically 2-3 times per week. Points represent the mean of tumor volume in $\mathrm{mm}^{3}$ of several tumors $(n=5)$ during the course of the treatment period. (B) Tumor volume on the last day of the experiment (64 days after tumor injection) is represented as a bar graph. ${ }^{* *} \mathrm{P}<0.01$ indicates statistically significant differences between experimental groups.

MPAP confers radiosensitizing effect on xenograft tumor growth. To assess the antitumor effect of MPAP with or without radiation in vivo, we established xenografts of A549 cells in nude mice. As shown in Fig. 5, treatment with MPAP slightly reduced the tumor size, but this was not statistically significant.

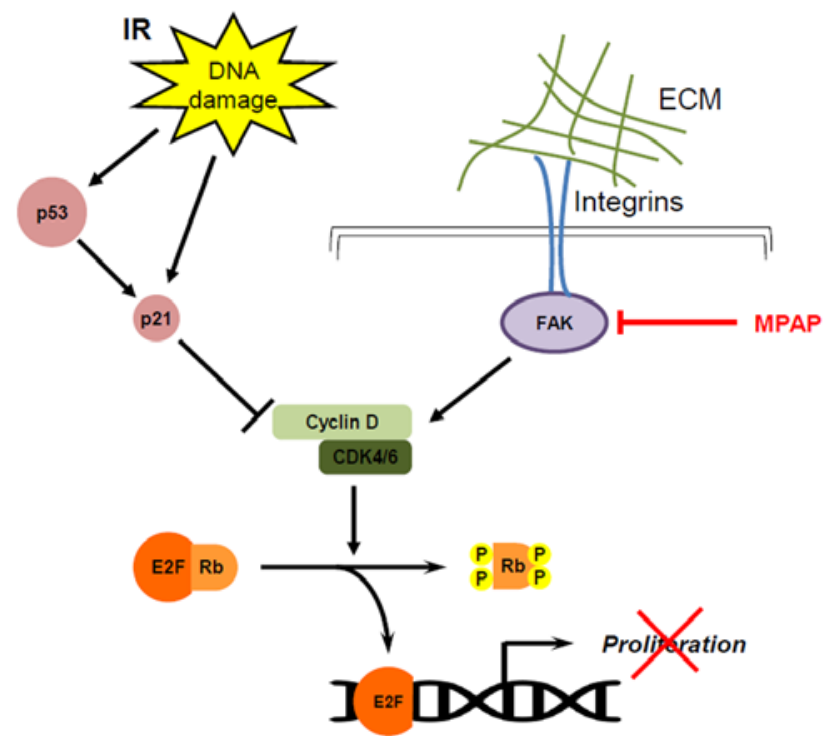

G1 $\quad$ s

Figure 6. Schematic diagram of the signaling pathway by which combination treatment with MPAP and ionizing radiation control cell cycle arrest. Ionizing radiation activates checkpoint pathways to repair or induce apoptosis in DNA-damaged cells. In contrast, FAK1 expression induced by integrin-mediated signaling progresses the cell cycle from $\mathrm{G} 1$ to $\mathrm{S}$ phase through cyclin D activation and RB phosphorylation. A newly synthesized FAK1 inhibitor, MPAP, effectively induced G1 phase arrest by inhibiting both FAK1 activity and RB phosphorylation

However, 5 Gy of IR resulted in significant tumor reduction. Combined treatment suppressed tumor growth better than the IR alone. These data suggest that MPAP efficiently contributes to radiation-mediated tumor growth reduction.

\section{Discussion}

Generally, radiation therapy is regarded as the most effective therapeutic approach for cancer treatment and it exhibits some benefits when compared with chemotherapy (25). However, radioresistance and recurrence are major limitations in the long-term survival of patients undergoing radiation therapy (26). In addition, radiotherapy can cause various side-effects, such as fatigue, nausea, vomiting, appetite loss, emotional changes, hair loss, and in particular, tissue fibrosis. Thus, a novel therapeutic approach that reduces these sideeffects would improve radiotherapy. As a possible approach, we propose a combination treatment of a reduced dose of ionizing radiation along with a radiosensitizer, such as MPAP. As we mentioned earlier, several compounds that inhibit FAK activation have been developed by pharmaceutical companies. The mother compound of MPAP, TAE226 has been previously reported to possess cytotoxic activity against cancer cells. However, TAE226 exhibited radiosensitizing effects on only two out of seven glioblastoma cell lines at $10 \mu \mathrm{M}$, and exhibited no such effects on lung, pancreatic, or colorectal cancer cells $(27,28)$. Therefore, the advantages of MPAP are its lower $\mathrm{IC}_{50}$ value against FAK than that of TAE226, as well as its radiosensitizing effect on lung cancer cells, which is not exhibited by TAE226. 
In this study, we showed that MPAP could inhibit cancer cell proliferation and the phosphorylation of FAK1 at Tyr397, Tyr576/577 and Tyr925 (Fig. 1). Moreover, the combination treatment of MPAP and IR suppressed cancer cell proliferation in wild-type p53 cells, and this effect was even higher in a p53-null background (Fig. 2). Several studies have demonstrated that FAK1 inhibits the transcriptional activity of $\mathrm{p} 53$ through protein-protein interactions, and that p53 also binds to the FAK1 promoter, inhibiting its activity. This feedback mechanism is well-correlated with the overexpression or upregulation of FAK1 in p53-mutated cancers (29). Furthermore, mutation or deletion of the p53 gene is the most frequent genetic change in human cancer. Therefore, combination therapy with both a FAK1 inhibitor and radiation could represent a significant advance in therapeutic strategy.

Radiation can induce apoptotic cell death or cell cycle arrest to suppress tumor cell survival. Our data showed that 4 Gy of radiation decreased long-term clonogenic survival but did not alter cell cycle progression according to short-term analysis.

While the induction of cell cycle arrest by IR was not specifically observed in this study, this could have simply been undetected, owing to the fact that we did not perform a time-kinetic analysis of cell cycle perturbations or investigate the synchronization of cells. The function of MPAP per se on cell cycle distributions differed in the two cell lines, leading to S and G2/M arrest in A549 cells and G1 arrest in H1299 cells. Although FAK inhibition has been reported to reduce the amount of cyclin D1 and arrest cells in the G1/S phase (30), cell cycle accumulation in the G1, S or G2 phases is dependent on cell type, radiation dose, harvest time after IR, and the method of FAK inhibition, such as gene deletion, ATP-binding site blocker or multi-kinase inhibitor. This is supported by the finding that hepatocellular carcinoma cells transduced with FAK shRNA exhibit G2/M arrest, whereas an inhibitor of Tyr397 phosphorylation of FAK (PND-1186) induces G1 arrest under the same conditions, suggesting that the signaling pathways triggered by FAK kinase activity could be distinct from the scaffolding function of FAK (31). In addition, TAE226 induces G2 arrest in glioma cells and increases apoptosis (23). However, MPAP significantly enhanced G1 cell cycle arrest when used in combination with IR in A549 cells, and did so more significantly in H1299 cells (Fig. 3). G1/S cell cycle progression is controlled by the phosphorylation of RB, and the cyclin D1/CDK4 complex is responsible for this regulation (32). Several studies have demonstrated that integrin-mediated cell adhesion controls cell cycle progression by regulating the expression levels and activities of cyclins, CDKs and CDK inhibitors $(33,34)$. The activation of FAK1 by integrins accelerates the G1 to S-phase cell cycle progression primarily by increasing cyclin D1 expression directly at the transcriptional level in a p21-independent manner (30). Consistent with previous reports, the most distinctive changes were observed in RB and $\mathrm{pRB}$ expression by MPAP/IR treatment in both cell lines. In addition, the fact that the decrease in cyclin D1 and CDK4 expression mediated by MPAP/IR was clearly shown in p53 null-type H1299 cells, compared with the mild inhibition in p53 wild-type A549 cells (Fig. 4). These results suggest that MPAP/IR-mediated G1 arrest is independent of p53 status and the alteration of the cyclin D1/CDK4/pRB axis could be involved in MPAPmediated radiosensitization. Under genotoxic conditions such as IR, diverse DNA damage lesions trigger the activation of multiple intracellular signaling pathways, resulting in cell cycle arrest, DNA repair, or apoptosis. Numerous studies have demonstrated that p53/p21 are involved in G1 as well as $\mathrm{G} 2 / \mathrm{M}$ arrest after irradiation, thereby sensitizing tumor cells to radiation (35-37). As expected, IR markedly increased levels of the p53 and p21 proteins in p53 wild-type cells, but the induction of cyclin D1/cdk4 and reduction of $\mathrm{pRB}$ were concurrently observed. These data suggest that diverse signaling pathways are involved in the regulation of cell cycle progression. In p53-null H1299 cells, p21 expression was markedly increased by the combination treatment of MPAP and IR, and these increased levels of p21 might participate in the G1 cell cycle arrest. The function of p21 can confer sensitivity to IR, as permanent, replicative death can result from prolonged G1 arrest (38). Even though p53 is not the only transcription factor to regulate $\mathrm{p} 21$ expression, it remains unknown how p21 protein levels are elevated under p53-null conditions, and this is worth investigating in the future.

Finally, the radiosensitizing effect of MPAP was determined in tumor-transplanted mice. Owing to the powerful antitumor effect of MPAP itself at doses above $0.5 \mathrm{mg} / \mathrm{kg}$, similar to that of IR, we did not observe a synergistic effect of the combination treatment of MPAP and IR (data not shown). When a low dose of MPAP $(10 \mu \mathrm{g} / \mathrm{kg})$ was administered as a single dose with IR, the tumor burden was more efficiently suppressed than by either MPAP or IR alone (Fig. 5). Overall, these results demonstrate that the combination treatment of MPAP and IR can efficiently suppress lung tumor growth by the induction of G1 arrest via the regulation of RB phosphorylation in a p53-independent manner (Fig. 6). Considering that the detailed mechanism of the radiosensitizing effects of MPAP remains to be clarified, our results provide insights for the development of an effective multi-targeted approach to the treatment of malignant tumors that exhibit a p53-null background.

\section{Acknowledgements}

The present study was supported by a grant from the Korea Institute of Radiological and Medical Sciences (KIRAMS), funded by Ministry of Science, ICT and Future Planning (grant nos. 1711045557, 1711045538, 1711045554 and 50531-2017), and in part by grant no. 20131610101840 from the Ministry of Trade, Industry \& Energy, Republic of Korea.

\section{References}

1. Parsons JT: Focal adhesion kinase: The first ten years. J Cell Sci 116: 1409-1416, 2003.

2. Mitra SK, Hanson DA and Schlaepfer DD: Focal adhesion kinase: In command and control of cell motility. Nat Rev Mol Cell Biol 6: 56-68, 2005.

3. Hanks SK, Calalb MB, Harper MC and Patel SK: Focal adhesion protein-tyrosine kinase phosphorylated in response to cell attachment to fibronectin. Proc Natl Acad Sci USA 89: 8487-8491, 1992.

4. Calalb MB, Polte TR and Hanks SK: Tyrosine phosphorylation of focal adhesion kinase at sites in the catalytic domain regulates kinase activity: A role for Src family kinases. Mol Cell Biol 15: 954-963, 1995. 
5. Owen JD, Ruest PJ, Fry DW and Hanks SK: Induced focal adhesion kinase (FAK) expression in FAK-null cells enhances cell spreading and migration requiring both auto- and activation loop phosphorylation sites and inhibits adhesion-dependent tyrosine phosphorylation of Pyk2. Mol Cell Biol 19: 4806-4818, 1999.

6. Sulzmaier FJ, Jean C and Schlaepfer DD: FAK in cancer: Mechanistic findings and clinical applications. Nat Rev Cancer 14: 598-610, 2014

7. Cancer Genome Atlas Network: Comprehensive molecular portraits of human breast tumours. Nature 490: 61-70, 2012.

8. Bell D, Berchuck A, Birrer M, Chien J, Cramer DW, Dao F, Dhir R, DiSaia P, Gabra H, Glenn P, et al; Cancer Genome Atlas Research Network: Integrated genomic analyses of ovarian carcinoma. Nature 474: 609-615, 2011.

9. Sood AK, Armaiz-Pena GN, Halder J, Nick AM, Stone RL, Hu W, Carroll AR, Spannuth WA, Deavers MT, Allen JK, et al: Adrenergic modulation of focal adhesion kinase protects human ovarian cancer cells from anoikis. J Clin Invest 120: 1515-1523, 2010.

10. van Nimwegen MJ and van de Water B: Focal adhesion kinase: A potential target in cancer therapy. Biochem Pharmacol 73 597-609, 2007

11. Siesser PMF and Hanks SK: The signaling and biological implications of FAK overexpression in cancer. Clin Cancer Res 12 3233-3237, 2006.

12. Ward KK, Tancioni I, Lawson C, Miller NL, Jean C, Chen XL, Uryu S, Kim J, Tarin D, Stupack DG, et al: Inhibition of focal adhesion kinase (FAK) activity prevents anchorage-independent ovarian carcinoma cell growth and tumor progression. Clin Exp Metastasis 30: 579-594, 2013.

13. Chen XL, Nam JO, Jean C, Lawson C, Walsh CT, Goka E, Lim ST, Tomar A, Tancioni I, Uryu S, et al: VEGF-induced vascular permeability is mediated by FAK. Dev Cell 22: 146-157, 2012.

14. Walsh C, Tanjoni I, Uryu S, Tomar A, Nam JO, Luo H, Phillips A, Patel N, Kwok C, McMahon G, et al: Oral delivery of PND-1186 FAK inhibitor decreases tumor growth and spontaneous breast to lung metastasis in pre-clinical models. Cancer Biol Ther 9 778-790, 2010.

15. Jean C, Chen XL, Nam JO, Tancioni I, Uryu S, Lawson C, Ward KK, Walsh CT, Miller NL, Ghassemian M, et al: Inhibition of endothelial FAK activity prevents tumor metastasis by enhancing barrier function. J Cell Biol 204: 247-263, 2014

16. Cabrita MA, Jones LM, Quizi JL, Sabourin LA, McKay BC and Addison CL: Focal adhesion kinase inhibitors are potent antiangiogenic agents. Mol Oncol 5: 517-526, 2011.

17. Halder J, Lin YG, Merritt WM, Spannuth WA, Nick AM, Honda T, Kamat AA, Han LY, Kim TJ, Lu C, et al: Therapeutic efficacy of a novel focal adhesion kinase inhibitor TAE226 in ovarian carcinoma. Cancer Res 67: 10976-10983, 2007.

18. Stokes JB, Adair SJ, Slack-Davis JK, Walters DM, Tilghman RW, Hershey ED, Lowrey B, Thomas KS, Bouton AH, Hwang RF, et al: Inhibition of focal adhesion kinase by PF-562,271 inhibits the growth and metastasis of pancreatic cancer concomitant with altering the tumor microenvironment. Mol Cancer Ther 10: 2135-2145, 2011

19. Wendt MK and Schiemann WP: Therapeutic targeting of the focal adhesion complex prevents oncogenic TGF-beta signaling and metastasis. Breast Cancer Res 11: R68, 2009.

20. Slack-Davis JK, Hershey ED, Theodorescu D, Frierson HF and Parsons JT: Differential requirement for focal adhesion kinase signaling in cancer progression in the transgenic adenocarcinoma of mouse prostate model. Mol Cancer Ther 8: 2470-2477, 2009 .
21. Yoon H, Dehart JP, Murphy JM and Lim ST: Understanding the roles of FAK in cancer: Inhibitors, genetic models, and new insights. J Histochem Cytochem 63: 114-128, 2015.

22. Liu TJ, LaFortune T, Honda T, Ohmori O, Hatakeyama S, Meyer T, Jackson D, de Groot J and Yung WK: Inhibition of both focal adhesion kinase and insulin-like growth factor-I receptor kinase suppresses glioma proliferation in vitro and in vivo. Mol Cancer Ther 6: 1357-1367, 2007.

23. Shi Q, Hjelmeland AB, Keir ST, Song L, Wickman S, Jackson D, Ohmori O, Bigner DD, Friedman HS and Rich JN: A novel lowmolecular weight inhibitor of focal adhesion kinase, TAE226, inhibits glioma growth. Mol Carcinog 46: 488-496, 2007.

24. Nam KY, Jin DH, No KT and Ahn SK: Discovery of FAK inhibitors using structure based drug design. Bull Korean Chem Soc 35: 3156-3157, 2014

25. Amin NP, Sher DJ and Konski AA: Systematic review of the cost effectiveness of radiation therapy for prostate cancer from 2003 to 2013. Appl Health Econ Health Policy 12: 391-408, 2014.

26. Begg AC, Stewart FA and Vens C: Strategies to improve radiotherapy with targeted drugs. Nat Rev Cancer 11: 239-253, 2011.

27. Hehlgans S, Lange I, Eke I and Cordes N: 3D cell cultures of human head and neck squamous cell carcinoma cells are radiosensitized by the focal adhesion kinase inhibitor TAE226. Radiother Oncol 92: 371-378, 2009.

28. Storch K, Sagerer A and Cordes N: Cytotoxic and radiosensitizing effects of FAK targeting in human glioblastoma cells in vitro. Oncol Rep 33: 2009-2016, 2015.

29. Golubovskaya VM and Cance WG: FAK and 553 protein interactions. Anticancer Agents Med Chem 11: 617-619, 2011.

30. Zhao J, Pestell R and Guan JL: Transcriptional activation of cyclin D1 promoter by FAK contributes to cell cycle progression. Mol Biol Cell 12: 4066-4077, 2001.

31. Gnani D, Romito I, Artuso S, Chierici M, De Stefanis C, Panera N, Crudele A, Ceccarelli S, Carcarino E, D'Oria V, et al: Focal adhesion kinase depletion reduces human hepatocellular carcinoma growth by repressing enhancer of zeste homolog 2 . Cell Death Differ 24: 889-902, 2017.

32. Giacinti $\mathrm{C}$ and Giordano A: RB and cell cycle progression. Oncogene 25: 5220-5227, 2006.

33. Assoian RK: Anchorage-dependent cell cycle progression. J Cell Biol 136: 1-4, 1997.

34. Assoian RK and Schwartz MA: Coordinate signaling by integrins and receptor tyrosine kinases in the regulation of G1 phase cellcycle progression. Curr Opin Genet Dev 11: 48-53, 2001.

35. Iliakis G, Wang Y, Guan J and Wang H: DNA damage checkpoint control in cells exposed to ionizing radiation. Oncogene 22: 5834-5847, 2003.

36. Stewart N, Hicks GG, Paraskevas F and Mowat M: Evidence for a second cell cycle block at G2/M by p53. Oncogene 10: 109-115, 1995.

37. Siles E, Villalobos M, Valenzuela MT, Núñez MI, Gordon A, McMillan TJ, Pedraza V and Ruiz de Almodóvar JM: Relationship between p53 status and radiosensitivity in human tumour cell lines. Br J Cancer 73: 581-588, 1996.

38. Waldman T, Zhang Y, Dillehay L, Yu J, Kinzler K, Vogelstein B and Williams J: Cell-cycle arrest versus cell death in cancer therapy. Nat Med 3: 1034-1036, 1997. 